\title{
Amino Acid Chelated Iron versus Ferrous Fumarate in the Treatment of Iron Deficiency Anemia with Pregnancy : Randomized Controlled Trial
}

\author{
Original \\ Article Ahmed Khairy Makled, Wessam Magdy Abuelghar, Ahmed AbdElshafy \\ El-Shahawy, Manar Abd Elmaksoud Elshazely* \\ Department of Obstetrics and Gynecology, Faculty of Medicine, Ain-Shams University, Cairo, \\ Egypt
}

\begin{abstract}
Background: Anemia is a major health problem. Iron deficiency is the most common cause of anemia during pregnancy. Suboptimal iron content in the average mother's diet and the presence of insufficient iron stores during the reproductive years are causes for this predominance. In many developing countries, iron deficiency anemia (IDA) in pregnancy is more of a rule than an exception with a prevalence of $52 \%$. In the Western societies, the frequency of IDA is approximately $25 \%$ in pregnant women not taking iron supplements and less than $5 \%$ in women taking iron supplements. Anemia has a significant impact on the health of the fetus and the mother. It can be associated with increased preterm labour, preeclampsia, and maternal sepsis. It can also lead to fetal loss or even perinatal deaths.

Aim: To find out whether there is a difference between amino acid chelated iron and iron salts (ferrous fumarate) in effect, safety, adverse effects and outcomes in treatment of iron deficiency anemia during pregnancy.

Patients and Methods: The study was conducted on pregnant women attending the antenatal care in the outpatient clinic at Ain-Shams University Maternity Hospital in the period from February 2019 to July 2019, with diagnosis of iron deficiency anemia between $14-18$ weeks with hemoglobin (HB) level 8-10.5g/dL, and serum ferritin $<15 \mu \mathrm{g} / 1$. One hundred fifty pregnant women who met the criteria were randomized in to 2 groups (iron chelated amino acid "IAAC" group and ferrous fumarate "FF" group). Hemoglobin level, blood indices, serum iron and serum ferritin levels were measured in both groups at $4,8,12$ weeks of treatment.

Results: There was a significant hematological improvement (mean HB level, blood indices, serum iron and ferritin levels) in both groups which was significantly higher in IAAC group.

Conclusion: Iron amino acid chelate achieves faster cure rate than ferrous fumarate in women with iron deficiency anemia in pregnancy as regards mean HB and serum ferritin level. Iron amino acid chelate is associated with fewer side effects than ferrous fumarate.
\end{abstract}

Key Words: Hemoglobin, gastrointestinal, iron deficiency anemia

Received: 23 October 2019, Accepted: 24 November 2019

Corresponding Author: Manar Abd Elmaksoud Elshazely, Department of Obstetrics and Gynecology, Faculty of Medicine, Ain-Shams University, Cairo, Egypt, Tel.: 01069491030, E-mail: manarelshazly407@gmail.com

ISSN: 2090-7625, February 2020, Vol.10, No. 1

\section{INTRODUCTION}

Anemia is characterized by reductions in hemoglobin concentration, red-cell count, or packed-cell. The mean minimum acceptable hemoglobin level during pregnancy by World Health Organization criteria is taken to be $11 \mathrm{~g} / \mathrm{dL}^{[1]}$.

Anemia is a common problem in obstetrics and perinatal care. The main cause of anemia in obstetrics is iron deficiency, which has a worldwide prevalence $20 \%-80 \%$ and consists of a primarily female population $^{[2]}$.
Maternal risks include increased risk of infection, depleted blood reserves during delivery and thus an increased risk of allogeneic blood transfusion in the case of significant blood loss, cardiovascular stress, anaemic symptoms (fatigue, reduced physical and mental capacities, headaches, orthostatic dizziness, exhaustion, etc.), prolonged hospitalization, decreased milk production in the puerperium, increased risk of post-natal depression, depleted maternal iron stores postpartum ${ }^{[2]}$.

For these reasons, the efficient treatment of anaemia has a positive impact on maternal as well as foetal outcomes ${ }^{[3]}$. 
The choice of treatment depends on the cause of the anaemia. Oral iron products or intravenous iron products can be used for iron therapy in cases of iron deficiency anemia (IDA). Various studies have shown that, once indicated, intravenous iron therapy is superior to oral iron therapy in terms of speed and absolute extent of haemoglobin increase $\mathrm{e}^{[4]}$.

The primary treatment for mild cases of iron-deficiency anaemia and iron deficiency without anaemia in pregnancy is per oral iron therapy (iron II salts or iron III polymaltose) at doses of 160-200 mg/day (ideally on an empty stomach, fractionated). The same applies to iron deficiency and depleted iron stores (ferritin $<30 \mu \mathrm{g} / \mathrm{L}$ ) without anaemia at the beginning of pregnancy, because of the additional requirement for iron in the course of the pregnancy. In the following clinical situations intravenous iron therapy is indicated in pregnancy from the second trimester onwards: lack of response to oral iron (Hb levels rising by less than $10 \mathrm{~g} / \mathrm{L}$ within 14 days), intolerance of oral iron products (gastrointestinal side effects) or lack of compliance, severe or advanced anaemia $(\mathrm{Hb}<9 \mathrm{~g} / \mathrm{dL})$, need for rapid and efficient anaemia treatment (advanced gestational age, placenta previa, etc. $)^{[5]}$.

It is a well-established fact that there is a physiological drop in hemoglobin $(\mathrm{Hb})$ in the mid trimester. This physiological drop is attributed to increase of plasma volume and hence decrease of blood viscosity ${ }^{[6]}$.

It's suggested that the lowest critical hemoglobin value in iron-treated pregnant women should be $110 \mathrm{~g} / 1 \quad(6.8 \mathrm{mmol} / \mathrm{L})$ in the $1^{\text {st }}$ trimester, and $105 \mathrm{~g} / \mathrm{L}(6.5 \mathrm{mmol} / \mathrm{L})$ in the $2^{\text {nd }}$ and $3^{\text {rd }}$ trimester ${ }^{[7]}$.

Oral iron preparations for correction of iron deficiency include iron salts, iron chelates and ferric hydroxide complexes. Ferrous salts are characterized by a variable absorption rate ${ }^{[8]}$.

Furthermore, gastrointestinal (GIT) disturbances occur in about $50 \%$ of patients taking these forms of oral iron preparations $^{[9]}$.

Amino acid chelated iron therapy was reported to have more rapid effect with less GIT side effects ${ }^{[8]}$ and this is what we want to prove in this study.

The journey of multi-amino acids chelated minerals during absorption pass through 3 stations ; amino acid cage catches the mineral through high quality organic legends, not affected by HCL. Then amino acid cage carries the mineral through 18 essential amino acids to protect it from anti-nutritional factors. After that the amino acid cage passes the minerals to the blood stream up to $95 \%$ absorption with minimal side effects.
This study aimed to compare between an iron amino acid chelated preparation and a conventional iron salt (ferrous fumarate) regarding the efficacy in correcting iron deficiency anemia with pregnancy and their tolerability.

\section{AIM OF THE WORK}

To compare between amino acid chelated iron and iron salts (ferrous fumarate) as regard effect, safety and adverse effects in treatment of iron deficiency anemia during pregnancy.

\section{PATIENTS AND METHODS}

Study design: This study was randomized controlled trial.

Setting: The study was conducted on the pregnant women who were attending the antenatal care in the outpatient clinic at Ain-Shams University Maternity Hospital in the period from February 2019 to July 2019.

Population of the study: Patients were recruited from the pregnant women who were attending the outpatient clinic at Ain-Shams University Maternity Hospital with the diagnosis of iron deficiency anemia between 14-18 weeks with hemoglobin level $8-10.5 \mathrm{~g} / \mathrm{dL}$ and serum ferritin $<15 \mu \mathrm{g} / \mathrm{l}$. The study protocol and patient informed consent were reviewed and approved by the Ethics Committee of the Obstetrics and Gynecology Department of Ain-Shams University.

Inclusion criteria: Pregnant women at 14-18 weeks of gestation calculated from the first day of their last menstrual period and confirmed or modified by ultrasound. Hemoglobin level 8-10.5 g/dL. Serum ferritin $<15 \mu \mathrm{g} / \mathrm{L}$.

Exclusion criteria: The presence of anemia from causes other than iron deficiency (e.g: folate deficiency anemia, vitamin B12 deficiency, thalassemia, etc...) and multiple gestations. The presence of clinical and/or laboratory evidence of hepatic, renal, hematological or cardiovascular disease. Patients with known gastritis. History of mal-absorption syndrome. Those with known hypersensitivity to iron preparations. Those under current iron supplementation.

\section{Randomization and allocation:}

Method of randomization: 150 Patients who fulfilled the criteria were randomized used random sequence computer generated list in such a way that every patient had equal chance to be among any of the two groups (1:1).

Allocation concealment was done using: Sequential, Numbered, Opaque Sealed (SNOS) envelopes. Each 
envelope contained one of the two types of iron used in our study and it was opened just before giving the treatment.

Sample size justification: The required sample size had been calculated using the IBM(C) SamplePower(C) software version 3.0.1 (IBM(C) Corp., Armonk, NY). The primary outcome measure was the difference between the 2 groups as regards the increase in hemoglobin at 4 weeks, 8 weeks and 12 weeks of treatment. A previous study reported that after 4 weeks, the hemoglobin level increased by $0.77 \pm 0.43 \mathrm{~g} / \mathrm{dl}$ versus $0.31 \pm 0.31 \mathrm{~g} / \mathrm{dl}$ in patients receiving IAAC or $\mathrm{FF}$, respectively. After 8 weeks, the hemoglobin level increased by $1.51 \pm 0.61 \mathrm{~g} / \mathrm{dl}$ versus $0.64 \pm 0.51 \mathrm{~g} / \mathrm{dl}$ and after 12 weeks, the hemoglobin level increased by $1.6 \pm 0.6 \mathrm{~g} / \mathrm{dl}$ versus $1.32 \pm 0.55 \mathrm{~g} /$ $\mathrm{dl}$, respectively ${ }^{[8]}$. So, it is estimated that a sample size of 68 patients in either study group was had a power of $80 \%$ to detect a statistically significant difference of $0.28 \mathrm{~g} / \mathrm{dl}$ between the 2 groups as regards the increase in hemoglobin at 12 weeks (corresponding to means of 1.6 versus $1.32 \mathrm{~g} / \mathrm{dl}$ ) with assumed common SD of $0.58 \mathrm{~g} / \mathrm{dl}$ (based on SD estimates of 0.60 and 0.55 ). The power for detecting a difference in the increase in hemoglobin at 4 weeks and 8 weeks was exceeding $99.9 \%$. These calculations used a two-sided unpaired test with a type I error of 0.05 . Assuming an attrition (drop-out) rate of $10 \%$, a total of 150 patients were recruited ( 75 patients per group).

Study intervention: All women who met the inclusion criteria were assessed:

Detailed history: Demographic factors like age and parity were recorded, detailed history of any medical condition. Gestational age was determined from last menstrual period or previous ultrasound scanning report

Physical examination: General examination including pulse, blood pressure, temperature, body weight, auscultation of lungs and heart. Abdominal examination: for fundal level.

Investigation: Baseline hemoglobin, hematocrit level, $\mathrm{MCV}, \mathrm{MCH}, \mathrm{MCHC}$, serum ferritin and serum iron were reported for every patient. Ultrasonography to confirm gestational age. CBC sample was performed by cell dyne 1800 device (Abbott laboratories USA), serum iron was estimated by the colorimetric method or ELISA kits. Ferritin was measured by ELISA kits or chemi-elumination method done in laboratories of Ain shams university maternity hospital. The 150 pregnant women were divided into 2 equal groups as follows ; Group A contained 75 pregnant women who were given iron chelated amino acid containing $15 \mathrm{mg}$ elemental iron (ferrotron) once daily (a dose recommended by the company of the product). Group B contained 75 pregnant women who were given take iron salt e.g. ferrous fumarate (Hema- Caps) (ferrous fumarate $350 \mathrm{mg}$ with elemental iron $115 \mathrm{mg}$ ) once daily but in severe cases of iron deficiency anemia $(\mathrm{Hb}<9 \mathrm{~g} / \mathrm{dl})$ this dose was doubled as recommended by the company of the product (no doubling had been done in group A). Some instructions were given to all the pregnant women included in our study as follow: Iron tablets should be taken at night or at least 1 hour prior to food to maximize absorption. Substances that inhibit absorption such as tannins and milk should be avoided; but fruit juice containing ascorbic acid should be taken in conjunction with iron supplements to increase their absorption.

Follow up: Follow up was done for all the pregnant women included in our study at baseline and then at 4 weeks ( $1^{\text {st }}$ judgement), 8 and 12 weeks after treatment. At each visit brief history for outcomes and adverse effects were reviewed.

Initial judgment was made in the follow up visit on week 4 and it included assessment of patient as regard $\mathrm{CBC}$ and ferritin serum level and serum iron.

Primary outcome hemoglobin $(\mathrm{Hb})$ rise after 12 weeks of treatment, while the secondary outcome Hemoglobin $(\mathrm{Hb})$ rise after 4 and 8 weeks of treatment; and the prevalence of adverse effects in each group during the treatment period.

Ethical Considerations: This study was done after approval of the Ethical committee of the department of Obstetrics and Gynecology, Faculty of Medicine, Ain-Shams University. Informed consent was taken from all participants before recruitment in the study, and after explaining the purpose and procedures of the study. The investigator obtained the written, signed informed consent of each subject prior to performing any study specific procedures on the subject. The investigator retained the original signed informed consent form. All laboratory specimens, evaluation forms, reports, and other records that leave the site were not include unique personal to maintain subject confidentiality.

\section{Statistical Methods:}

Data was analyzed using IBMC SPSSC Statistics version 23 (IBMC Corp., Armonk, NY). Analysis was done on an intention to treat (ITT) basis. Normally distributed numerical data was presented as mean and SD, and skewed data as median and interquartile range. Qualitative data was presented as number and percentage. Comparison of normally distributed numerical data was done using the unpaired t test. Skewed data was compared using the Mann-Whitney test. Categorical data was compared using the chi-squared test or Fisher's exact test, if appropriate. $P$-value $<0.05$ was considered statistically significant. 
Efficacy of intervention was assessed by calculation of the odds ratio (OR), relative risk (RR), absolute risk reduction (ARR) and number needed to treat (NNT).

Statistical Package: Data was statistically described in terms of mean \pm standard deviation $( \pm \mathrm{SD})$, or frequencies (number of cases) and percentages when appropriate. Comparison of numerical variables between the study groups was done using Student $t$ test for independent samples. For comparing categorical data, Chi square (x2) test will be performed. Exact test was used instead when the expected frequency was less than 5. P values less than 0.05 was considered statistically significant. For participants who were attended the first and second follow up visits but missed the third one, the intention to treat analysis was applied, and hence, the results of the third visit were assumed to be the same as the second one. Patients were highly encouraged by the authors' team to strictly stick to the follow up dates so as to minimize the drop-out rate.

\section{RESULTS}

Table 1 showed the demographic data among the study groups. Tables 2 and 3 showed haemoglobin parameters in the 2 groups. Table 4 showed prevalence of anemia among the 2 studied groups. Table 5 showed maternal side effects in the 2 groups.

Table 1: Demographic characteristics among the studied groups

\begin{tabular}{|c|c|c|c|c|}
\hline Variables & Measures & $\begin{array}{l}\text { IAAC } \\
(\mathrm{N}=75)\end{array}$ & $\begin{array}{c}\mathrm{FF} \\
(\mathrm{N}=75)\end{array}$ & $P$ \\
\hline \multirow{3}{*}{$\begin{array}{l}\text { Age } \\
\text { (years) }\end{array}$} & Mean \pm SD & $28.3 \pm 4.0$ & $27.7 \pm 4.0$ & \multirow{3}{*}{$\wedge 0.417$} \\
\hline & & & & \\
\hline & Range & $19.0-37.0$ & $19.0-35.0$ & \\
\hline \multirow{3}{*}{$\begin{array}{l}\text { BMI } \\
(\mathrm{kg} / \mathrm{m} 2)\end{array}$} & Mean \pm SD & $25.4 \pm 2.5$ & $25.1 \pm 1.8$ & \multirow{3}{*}{$\wedge 0.387$} \\
\hline & & & & \\
\hline & Range & $19.0-31.6$ & $21.2-29.9$ & \\
\hline \multirow{2}{*}{$\begin{array}{l}\text { Parity } \\
(n, \%)\end{array}$} & Nulli & $27(36.0 \%)$ & $29(38.7 \%)$ & \multirow[b]{2}{*}{$\# 0.736$} \\
\hline & Multi & $48(64.0 \%)$ & $46(61.3 \%)$ & \\
\hline \multirow{2}{*}{$\begin{array}{l}\text { GA } \\
\text { (weeks) }\end{array}$} & Mean \pm SD & $16.1 \pm 0.8$ & $16.0 \pm 0.8$ & \multirow{2}{*}{${ }^{\wedge} 0.487$} \\
\hline & Range & $15.0-18.0$ & $14.0-18.0$ & \\
\hline
\end{tabular}

${ }^{\wedge}$ Independent t-test, \#Chi square test

Table 2: Hematologic parameters after 4,8 and 12 weeks of treatment in the two study groups

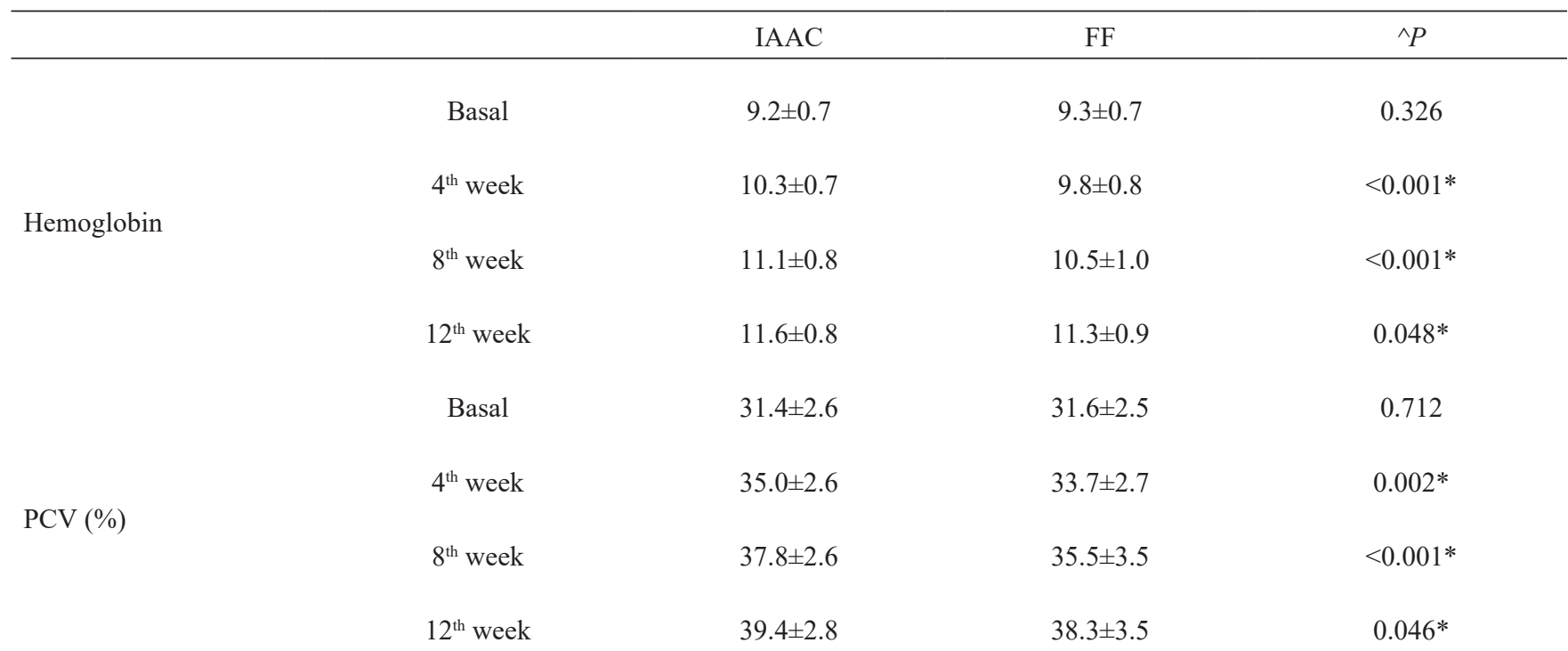


Makled et al.

\begin{tabular}{|c|c|c|c|c|}
\hline \multirow{4}{*}{ MCV (fL) } & Basal & $78.9 \pm 5.9$ & $79.8 \pm 5.9$ & 0.350 \\
\hline & $4^{\text {th }}$ week & $85.8 \pm 6.1$ & $82.0 \pm 6.3$ & $<0.001 *$ \\
\hline & $8^{\text {th }}$ week & $90.0 \pm 6.2$ & $73.6-103.2$ & $<0.001 *$ \\
\hline & $12^{\text {th }}$ week & $93.9 \pm 6.0$ & $91.5 \pm 7.9$ & $0.047 *$ \\
\hline \multirow{4}{*}{$\mathrm{MCH}(\mathrm{Pg})$} & Basal & $25.7 \pm 1.9$ & $26.0 \pm 2.0$ & 0.271 \\
\hline & $4^{\text {th }}$ week & $27.9 \pm 2.1$ & $26.9 \pm 2.2$ & $0.003 *$ \\
\hline & $8^{\text {th }}$ week & $28.8 \pm 2.0$ & $24.0-32.3$ & $0.001 *$ \\
\hline & $12^{\text {th }}$ week & $29.1 \pm 1.9$ & $28.3 \pm 2.3$ & $0.046^{*}$ \\
\hline \multirow{4}{*}{$\mathrm{MCHC}(\mathrm{g} / \mathrm{dL})$} & Basal & $29.2 \pm 0.8$ & $29.2 \pm 0.9$ & 0.840 \\
\hline & $4^{\text {th }}$ week & $29.5 \pm 0.8$ & $29.2 \pm 0.6$ & $0.003 *$ \\
\hline & $8^{\text {th }}$ week & $29.3 \pm 1.0$ & $29.5 \pm 1.1$ & 0.302 \\
\hline & $12^{\text {th }}$ week & $29.4 \pm 1.0$ & $29.5 \pm 1.0$ & 0.582 \\
\hline \multirow{4}{*}{ Serum iron $(\mu \mathrm{g} / \mathrm{dL})$} & Basal & $67.9 \pm 5.0$ & $68.7 \pm 4.9$ & 0.280 \\
\hline & $4^{\text {th }}$ week & $75.6 \pm 5.3$ & $71.4 \pm 5.7$ & $<0.001 *$ \\
\hline & $8^{\text {th }}$ week & $94.3 \pm 6.4$ & $795-1064$ & $<0001 *$ \\
\hline & $12^{\text {th }}$ week & $110.5 \pm 6.9$ & $107.3 \pm 9.2$ & $0.022 *$ \\
\hline \multirow{5}{*}{ Serum ferritin (ng/dL) } & Basal & $10.1 \pm 1.4$ & $10.3 \pm 1.7$ & 0.607 \\
\hline & $4^{\text {th }}$ week & $14.2 \pm 1.8$ & $12.6 \pm 1.7$ & $<0.001^{*}$ \\
\hline & & & & \\
\hline & $8^{\text {th }}$ week & $24.0 \pm 2.1$ & $18.8 \pm 2.4$ & $<0.001^{*}$ \\
\hline & $12^{\text {th }}$ week & $29.5 \pm 3.5$ & $28.2 \pm 3.2$ & $0.018^{*}$ \\
\hline
\end{tabular}

^Independent t-test, \#Paired t-test, *Significant, CI: Confidence interval

Table 3: Hemoglobin level difference from baseline in the two study groups

\begin{tabular}{lccc}
\hline Time & Measures & IAAC & FF \\
\hline $\begin{array}{l}\text { Change } \\
\text { At } 4^{\text {th }} \text { week }\end{array}$ & Mean \pm SD & $1.1 \pm 0.2$ & $0.5 \pm 0.2$ \\
& Range & $0.7-1.6$ & $0.2-1.0$ \\
Change & Mean \pm SD & $1.9 \pm 0.4$ & $1.1 \pm 0.6$ \\
At $8^{\text {th }}$ week & Range & $0.7-2.5$ & $0.0-2.7$
\end{tabular}




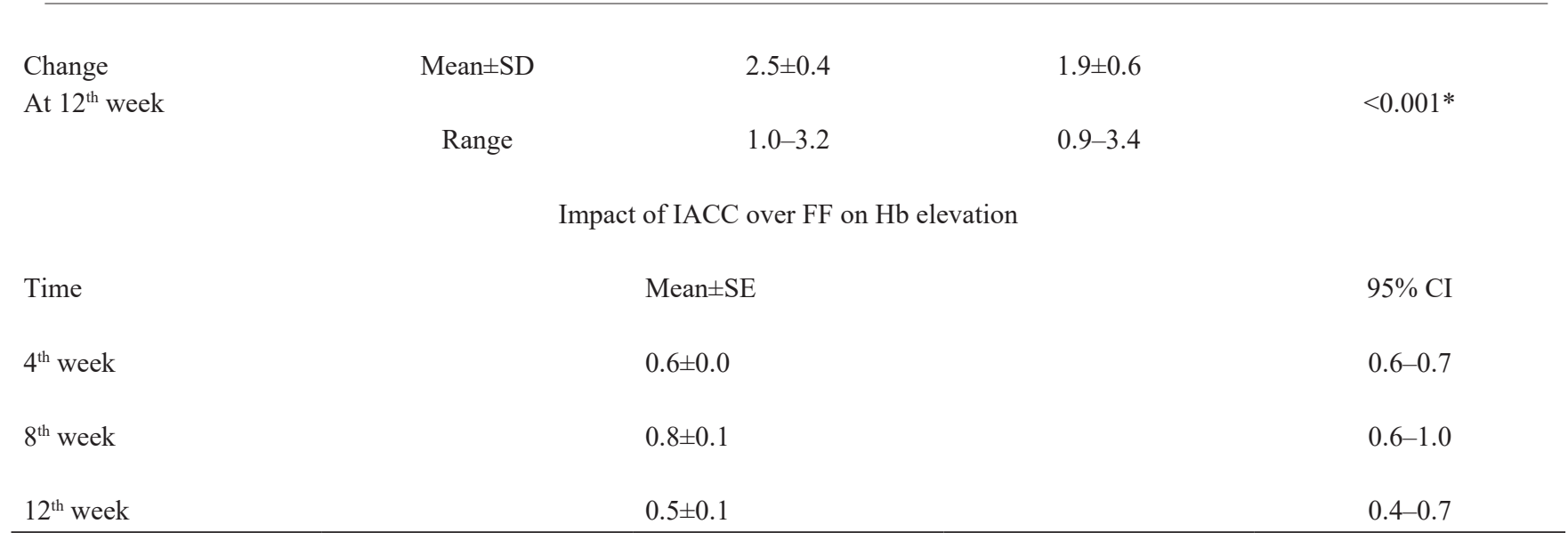

Table 4: Anemia ( $<11.0 \mathrm{gm} / \mathrm{dL})$ among the studied groups

\begin{tabular}{|c|c|c|c|c|}
\hline Time & IAAC & $\mathrm{FF}$ & $\wedge P$ & $\mathrm{RR}(95 \% \mathrm{CI})$ \\
\hline Basal & $75(100.0 \%)$ & $75(100.0 \%)$ & -- & \\
\hline $4^{\text {th }}$ week & $62(82.7 \%)$ & $68(90.7 \%)$ & 0.150 & $0.73(0.51-1.06)$ \\
\hline $8^{\text {th }}$ week & $28(38.4 \%)$ & $43(59.7 \%)$ & $0.010^{*}$ & $0.62(0.50-0.91)$ \\
\hline $12^{\text {th }}$ week & $11(15.5 \%)$ & $21(30.0 \%)$ & $0.040^{*}$ & $0.52(0.27-0.99)$ \\
\hline
\end{tabular}

\#Chi square test. *Significant

Table 5: Maternal side effects among the studied groups

\begin{tabular}{|c|c|c|c|c|}
\hline Findings & $\begin{array}{c}\text { IAAC } \\
(\mathrm{N}=75)\end{array}$ & $\begin{array}{c}\mathrm{FF} \\
(\mathrm{N}=75)\end{array}$ & $\wedge P$ & $\begin{array}{c}\mathrm{RR} \\
(95 \% \mathrm{CI})\end{array}$ \\
\hline Constipation & $19(25.3 \%)$ & $33(44.0 \%)$ & $0.016^{*}$ & $0.58(0.36-0.92)$ \\
\hline Abdominsl pain & $17(22.7 \%)$ & $35(46.7 \%)$ & $0.002 *$ & $0.49(0.30-0.79)$ \\
\hline Black stool & $14(18.7 \%)$ & $28(37.3 \%)$ & $0.011^{*}$ & $0.50(0.29-0.87)$ \\
\hline Gastric upset & $12(16.0 \%)$ & $27(36.0 \%)$ & $0.005^{*}$ & $0.44(0.24-0.81)$ \\
\hline Nausea & $8(10.7 \%)$ & $18(24.0 \%)$ & $0.031 *$ & $0.44(0.21-0.96)$ \\
\hline Vomiting & $4(5.3 \%)$ & $12(16.0 \%)$ & $0.034^{*}$ & $0.33(0.11-0.99)$ \\
\hline
\end{tabular}

${ }^{\wedge}$ Chi square test, RR: Relative risk, ${ }^{*}$ Significant, CI: Confidence interval 
Fig. 1: The mean hemoglobin level in both groups

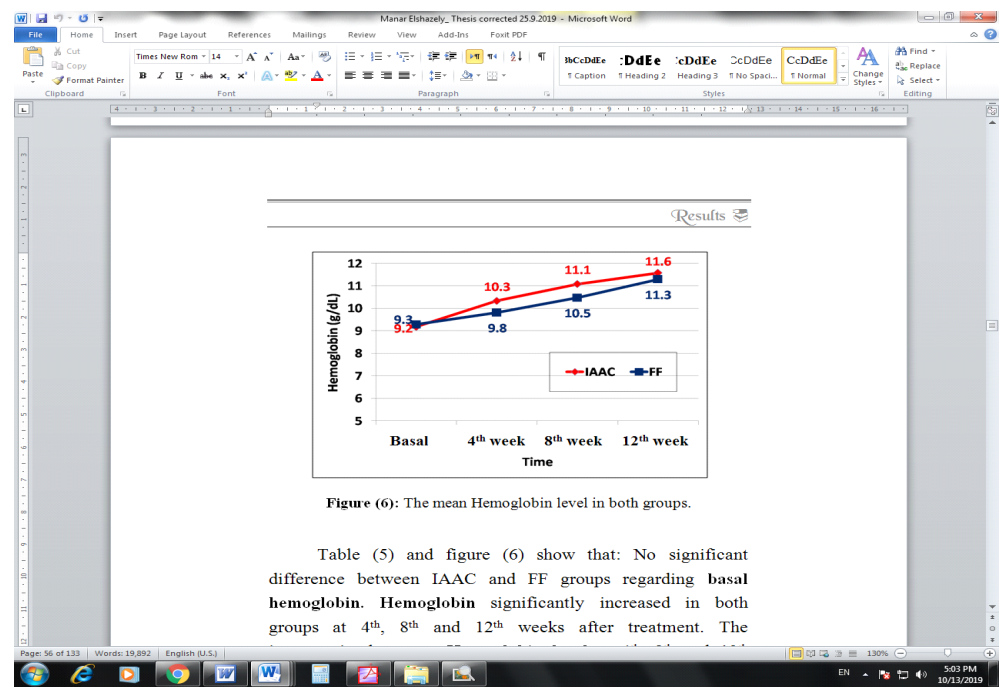

\section{DISCUSSION}

Anemia is the most common health problem affecting pregnant women in both developed and developing countries ${ }^{[10]}$. Oral iron is considered the first choice to correct IDA and replace iron stores as this allows the normal mechanism of absorption; beside its low cost and effectiveness ${ }^{[11]}$.

Oral iron preparations for the correction of iron deficiency include iron salts, iron chelates and ferric hydroxide complexes ${ }^{[8]}$. Ferrous salts are characterized by a variable absorption rate ${ }^{[12]}$. Furthermore, gastrointestinal (GIT) disturbances occur in about $50 \%$ of patients taking this form of oral iron preparations ${ }^{[9]}$. Amino acid chelated iron therapy was reported to have more rapid effect with less GIT side effects ${ }^{[13]}$.

In our controlled clinical trial conducted to compare IAAC containing $15 \mathrm{mg}$ of elemental iron and FF containing $350 \mathrm{mg}$ with elemental iron 115 mg, 168 women were recruited of which 15 did not meet the inclusion criteria, 3 refused to participate and one hundred fifty women were distributed into 2 groups each is 75 pregnant women attending the outpatient clinic at Ain-Shams University Maternity Hospital who are diagnosed as iron deficiency anemia between 14-18 weeks with hemoglobin level 8-10.5 g/dL and serum ferritin $<15 \mu \mathrm{g} / \mathrm{l}$. Group A received iron chelated amino acid while group B received ferrous fumarate. Follow up for outcomes was done by $\mathrm{CBC}$, serum ferritin level and serum iron level every 4 weeks for 12 weeks. Four patients were lost for follow up in group A; while 5 patients were lost in group B making the final analyzed patients : 71 and 70 in groups $\mathrm{A}$ and $\mathrm{B}$, respectively.

There was no statistically significant difference between both groups as regards demographic data.

There was no significant difference between IAAC and FF groups regarding basal hemoglobin. There was significant increase in mean $\mathrm{Hb}$ level in both groups at $4^{\text {th }}, 8^{\text {th }}$ and $12^{\text {th }}$ weeks after treatment. However, this was significantly higher in IAAC group than in FF group. Such that at 12 weeks of treatment; mean hemoglobin in IAAC group was $11.6 \pm 0.8$ while in FF group was $11.3 \pm 0.9$ and mean change in hemoglobin in IAAC group was $2.5 \pm 0.4$ while in FF group was $1.9 \pm 0.6$.

These results agree with the results of Abdel Moety et $a l .{ }^{[8]}$ who have shown that at the end of 12 weeks of treatment, the cure rate (percentage of women who reached the WHO hemoglobin target of $11 \mathrm{~g} / \mathrm{dL}$ ) was not significantly different between both groups. This means that from the therapeutic point of view, both IAAC and FF corrected iron deficiency anemia accompanying pregnancy. However, IAAC offered a significantly faster improvement rate of hemoglobin level when compared with FF.

These results are in agreement with the results of Szarfarc et al. ${ }^{[14]}$, who reported that both groups 
showed significant increase in HB level however ferrous bisglycinate was significantly more effective than ferrous sulphate even in lower doses. Furthermore, Kamdi and Palkar ${ }^{[15]}$ reported that IAAC resulted in a significantly higher rise in hemoglobin levels on day 14 and 28 when compared to ferrous ascorbate. Also, Singh S. et al. 2011, study on 375 adult female patients, 42 day daily treatment with ferrous bisglycinate or ferrous sulphate resulted in significant increase in hemoglobin $(\mathrm{Hb})$ levels. On day 42, the mean increase in $\mathrm{Hb}$ of the group receiving ferrous bisglycinate $(22.72 \%)$ was significantly higher than that of the group receiving ferrous sulphate (18.66\%).

Proportion of anemic patients at $8^{\text {th }}$ and $12^{\text {th }}$ weeks after treatment was significantly lower in IAAC group than in FF group.

As regards basal serum ferritin level, there was no significant difference between the two groups in our study. However, after treatment there was significant increase in serum ferritin level in both groups. At $12^{\text {th }}$ week; mean serum ferritin level in IAAC group was $29.5 \pm 3.5$ while in FF group was $28.2 \pm 3.2$ and the mean change in serum ferritin in IAAC group was $18.5 \pm 3.1$ while in FF group was 17.7 \pm 2.9 .

These results are in agreement with the results of Szarfarc et $a l^{[14]}$, who reported that ferrous bisglycinate group had a significant rise in serum ferritin. Also, Youssef et al., 2014 reported significant increase in serum ferritin level with ferrous bisglycinate than other iron preparations $(p<.05)$. Kamdi and Palkar ${ }^{[15]}$ reported that IAAC resulted in a significantly higher rise in ferritin levels on day 14 and 28 when compared to ferrous ascorbate.

In our study, there was no significant difference between IAAC and FF groups regarding basal PCV, $\mathrm{MCV}, \mathrm{MCH}, \mathrm{MCHC}$ and serum iron. PCV, MCV, $\mathrm{MCH}, \mathrm{MCHC}$ and serum iron significantly increased in both groups at $4^{\text {th }}, 8^{\text {th }}$ and $12^{\text {th }}$ weeks after treatment. $\mathrm{PCV}, \mathrm{MCV}, \mathrm{MCH}, \mathrm{MCHC}$, serum iron at $4^{\text {th }}, 8^{\text {th }}$ and $12^{\text {th }}$ weeks after treatment were significantly higher in IAAC group than in FF group. PCV, MCV, $\mathrm{MCH}, \mathrm{MCHC}$, serum iron elevation at $4^{\text {th }}, 8^{\text {th }}$ and $12^{\text {th }}$ weeks after treatment were significantly higher in IAAC group than in FF group.

In our study, maternal side effects were significantly less frequent in IAAC group than in FF group. Constipation was manifested in $19(25 \%)$ of patients in IAAC group and 33 (44\%) patients in FF group. Abdominal pain was manifested in $17(22.7 \%)$ patients in IAAC group and 35(46.7\%) patients in FF group. Black stool was manifested in $14(18.7 \%)$ patients in IAAC group and $28(37.3 \%)$ patients in FF group. Gastric upset was manifested in $12(16 \%)$ of patients in IAAC group and $27(36 \%)$ patients in FF group. Nausea was manifested in $8(10.7 \%)$ patients in IAAC group and $18(24 \%)$ patients in FF group. Vomiting was manifested in $4(5.3 \%)$ of patients in IAAC group and $12(16 \%)$ patients in $\mathrm{FF}$ group.

These results are in agreement with the results of Milman et al., 2014 who reported that gastrointestinal complaints were significantly less frequent in the bisglycinate group than in the sulfate group. The bisglycinate group also tended to have a lower frequency of black stools, indicating a lower fecal excretion of non-absorbed iron.

Maternal compliance was significantly more frequent in IAAC group than in FF group. The tolerability of amino acid chelated iron preparations in comparison to traditional iron salts was confirmed in a lot of previous studies ${ }^{[8,16]}$.

There was no significant correlation between hematological improvements and maternal age among the studied groups. There was no significant correlation between hematological improvements and gestational age (GA) among IAAC group. There was a significant correlation between hematological improvements except MCHC and GA among FF group.

\section{CONCLUSION}

Iron amino acid chelate achieve faster cure rate than ferrous fumarate in women with mid trimesteric iron deficiency anemia in pregnancy as regards mean HB and serum ferritin level. Iron amino acid chelates are associated with fewer side effects than ferrous fumarate.

\section{CONFLICT OF INTEREST}

There are no conflicts of interest.

\section{REFERENCES}

1. Jufar AH and Zewde T. Prevalence of anemia among pregnant women attending antenatal care at tikur anbessa specialized hospital, Addis Ababa Ethiopia. J Hematol Thromb Dis. 2014; 2(125):2

2. Breymann C. Iron deficiency anemia in pregnancy. In: seminars in hematology. WB Saunders, 2015; P. 339-347. 
3. Breymann C, Honegger C, Surbek D. Diagnosis and treatment of irondefciency anaemia in pregnancy, Arch Gynecol Obstet. 2017; 296:1229-1234.

4. Breymann C, Bian XM, Blanco-Capito LR, Chong C, Mahmud G, Rehma R. Expert recommendations for the diagnosis and treatment of irondeficiency anemia during pregnancy and the postpartum period in the Asia-Pacifc region. J Perinat Med. 2011; 39(2):113-121.

5. Breymann C, Honegger C, Holzgreve W, Surbek D. Diagnosis and treatment of iron-deficiency anaemia during pregnancy and postpartum. Archives of gynecology and obstetrics. 2010; 282(5):577-80.

6. Tan EK and Tan EL. Alterations in physiology and anatomy during pregnancy. Best practice \& research Clinical obstetrics \& gynaecology. $2013 ; 27(6): 791-802$.

7. Tabrizi FM and Barjasteh S. Maternal hemoglobin levels during pregnancy and their association with birth weight of neonates. Iranian journal of pediatric hematology and oncology. 2015; 5(4):211.

8. Abdel-Moety GA, Ali AM, Fouad R, Ramadan W, Belal DS, Haggag HM. Amino acid chelated iron versus an iron salt in the treatment of iron deficiency anemia with pregnancy: A randomized controlled study. European Journal of Obstetrics \& Gynecology and Reproductive Biology. 2017; 210:242-246.

9. Galy B, Ferring-Appel D, Sauer SW, Kaden S, Lyoumi $\mathrm{S}$, Puy $\mathrm{H}$, et al. Iron regulatory proteins secure mitochondrial iron sufficiency and function. Cell Metab 2010;12:194-201.
10. Bah A, Wegmuller R, Cerami C, Kendall L, Pasricha SR, Moore SE, Prentice AM. A double blind randomised controlled trial comparing standard dose of iron supplementation for pregnant women with two screen-and-treat approaches using hepcidin as a biomarker for ready and safe to receive iron. BMC Pregnancy Childbirth. 2016; 16(1):157.

11. Reveiz L, Gyte GM, Cuervo LG, Casasbuenas A. Treatments for iron deficiency anaemia in pregnancy. Cochrane database of systematic reviews. 2011(10).

12. Sharma N. "Iron absorption: IPC therapy is superior to conventional iron salts," Obstetrics \& Gynecology, 2001; pp. 515-519.

13. Burns D. Enhanced transport of amino acid chelated minerals. Research and Development. Concord, California: Seroyal Brands, Inc.; 2002.

14. Szarfarc SC, de Cassana LM, Fujimori E, GuerraShinohara EM, de Oliveira IM. Relative effectiveness of iron bis-glycinate chelate (Ferrochel) and ferrous sulfate in the control of iron deficiency in pregnant women. Arch Latinoam Nutr 2001; 51: 42-7.

15. Kamdi SP and Palkar PJ. Efficacy and safety of ferrous asparto glycinate in the management of iron deficiency anaemia in pregnant women. J Obstet Gynecol 2015;35(1):4-8.

16. Milman N, Jnsson L, Dyre P, Pedersen PL, Larsen LG. Ferrous bisglycinate $25 \mathrm{mg}$ iron is as effective as ferrous sulfate $50 \mathrm{mg}$ iron in the prophylaxis of iron deficiency and anemia during pregnancy in a randomized trial, J. Perinat. Med. 2014; 42(2): 197-206 